\title{
AVALIAÇÃO DO ESTADO NUTRICIONAL DOS IDOSOS RESIDENTES EM FORTALEZA/CE: O USO DE DIFERENTES INDICADORES ANTROPOMÉTRICOS
}

\author{
NUTRITIONAL STATUS ASSESSMENT OF ELDERLY RESIDENTS OF \\ FORTALEZA,CE, BRAZIL: THE USE OF DIFFERENT ANTHROPOMETRIC \\ INDICATORS
}

\begin{abstract}
RESUMO
O objetivo deste estudo foi avaliar, utilizando diferentes indicadores antropométricos, o estado nutricional dos idosos de Fortaleza. Este estudo é de base populacional, do tipo transversal, com coleta de dados primários. As variáveis antropométricas analisadas foram: índice de massa corporal (IMC), dobra cutânea tricipital (DCT) e circunferência muscular do braço (CMB). O estado nutricional foi definido a partir dos diagnósticos obtidos com a análise das variáveis antropométricas: eutrófico (idoso, no qual as três variáveis antropométricas (IMC, DCT e CMB), simultaneamente, indicassem o estado de eutrofia, segundo os padrões adotados) e não eutrófico (demais idosos). Foram selecionados 385 domicílios para comporem a amostra deste estudo, nos quais foram entrevistados 483 idosos (68\% mulheres). Quanto ao IMC, $47,3 \%$ do total de idosos foram considerados eutróficos. As mulheres apresentaram maior proporção de valores de IMC excessivo (21,9\%), quando comparadas aos homens (13,5\%). Foi verificada associação estatisticamente significativa entre adequação de IMC e sexo. Os valores de DCT mostraram que $54,4 \%$ do total de idosos eram eutróficos. Não houve associação estatisticamente significativa entre a adequação da DCT e sexo. Quanto à CMB, os homens apresentaram maior prevalência de desnutrição (66,5\%), quando comparados às mulheres (40,6\%). Foi verificada associação estatisticamente significativa entre adequação da CMB e sexo. Ao verificar o estado nutricional por meio das variáveis antropométricas, observou-se que $83,9 \%$ dos homens foram considerados não eutróficos, assim como maior parte das mulheres $(74,2 \%)$. Foi observada associação estatisticamente significativa entre estado nutricional e sexo. Os idosos de Fortaleza apresentam estado nutricional vulnerável, visto as prevalências de não eutróficos.
\end{abstract}

Palavras-chave: Idoso; Antropometria; Estado nutricional; Vigilância da população.

\section{ABSTRACT}

The aim of this study was to evaluate the anthropometric variables of elderly people in Fortaleza. This is a populational, cross-sectional household-based study with collection of primary data. The anthropometric variables analyzed were: body mass index (BMI), triceps skinfold thickness (TST) and arm muscle circumference (AMC). Nutritional status was defined from the anthropometrics variables and diagnosed as: eutrophic (elderly whose BMI,TST and AMC simultaneous measures was identified as eutrophic) and non-eutrophic (other elderly people). The sample for the study was composed of 385 selected dwellings, at which 483 elderly people were interviewed ( $68 \%$ of them women). As regards BMI, $47.3 \%$ of the elderly concerned were considered eutrophic. A higher proportion of the women had excessive BMI (21.9\%) than the men (13.5\%). There was a statistically significant association between BMI adequation and sex. The TST measurements indicated that $54.4 \%$ of the elderly were eutrophic. No statistically significant association was noted between TST adequation and sex. As for AMC, the men presented a higher prevalence of desnutrition (66.5\%) than the women (40.6\%). A statistically significant association between AMC adequation and sex was found. With respect to nutritional status, it was observed that $83.9 \%$ of the men and $74.2 \%$ of the women were classified as non-eutrophic. There was a statistically significant association between nutritional status and sex. The elderly population of Fortaleza exhibit a vulnerable nutritional status in view of the prevalence of non-eutrophic individuals.

Key words: Aged; Anthropometry; Nutritional status; Population surveillance.

1. Universidade Estadual da Paraíba. Departamento de Fisioterapia. Pesquisadora do Núcleo de Estudos e Pesquisas Epidemiológicas (NEPE), Campina Grande, PB. Brasil.

2. Universidade de São Paulo. Departamento de Epidemiologia da Faculdade de Saúde Pública, São Paulo, SP. Brasil.

3. Universidade de São Paulo. Departamento de Nutrição da Faculdade de Saúde Pública, São Paulo, SP. Brasil. 


\section{INTRODUÇÃO}

A antropometria tem se mostrado instrumento importante na avaliação do estado nutricional de indivíduos de vários grupos etários, pois proporciona informações sobre medidas físicas e composição corporal, além de ser um método de baixo custo, não invasivo e de fácil e rápida aplicação. Estudos epidemiológicos realizados com idosos têm utilizado variáveis antropométricas como forma de avaliar o estado nutricional ${ }^{1-6}$. Nesses estudos, as variáveis antropométricas comumente utilizadas são: peso, estatura, índice de massa corporal, perímetros (braço, panturrilha) e dobras cutâneas (tricipital, subescapular).

Estudos utilizando o índice de massa corporal (IMC) como forma de avaliar o estado nutricional de idosos, têm mostrado preocupantes prevalências de baixo peso ${ }^{7-10}$. Marucci e Barbosa $^{10}$, em pesquisa realizada com idosos residentes em São Paulo, verificaram que $24,1 \%$ dos idosos apresentavam baixo peso, tendo os homens maior prevalência $(29,2 \%)$ que as mulheres $(20,6 \%)$.

Com relação à dobra cutânea tricipital (DCT), estudos realizados com idosos mostram a tendência de diminuição dos valores com o avançar do grupo etário. Esses mesmos estudos mostram, ainda, que as mulheres apresentam maior reserva de gordura que os homens ${ }^{1,2,11-13}$. Martroeni ${ }^{13}$, em estudo com idosos residentes em Joinvile/SC, observou que maior parte dos idosos $(46,7 \%)$ apresentou valores adequados de DCT.

Quanto à reserva de massa muscular, estudos têm mostrado que os homens apresentam mais massa muscular que as mulheres, a qual tende a diminuir com o processo de envelhecimento. Delarue et al..$^{14} \mathrm{em}$ estudo realizado com idosos residentes em 2 localidades da França, utilizando a circunferência muscular do braço (CMB) como indicador de reserva de massa muscular, observaram que os valores de CMB dos idosos homens de ambas as localidades, foram maiores, quando comparados aos valores de CMB das mulheres. Tendência semelhante tem sido registrada em outros estudos ${ }^{1,2,12}$.

No Brasil, estudos epidemiológicos com idosos ainda são poucos ${ }^{15,16}$, assim como estudos antropométricos ${ }^{11-13,17}$, tornando desconhecida as características antropométricas dos idosos de algumas regiões do país. No Nordeste, informações epidemiológicas sobre a situação nutricional dos idosos, são reduzidas. Menezes ${ }^{8}$, avaliando o estado nutricional de idosos residentes em instituições de longa permanência de Fortaleza, observou que $49,8 \%$ dos idosos apresentavam baixo peso. Em termos populacionais, não foi encontrado na literatura especializada, informações sobre o estado nutricional dos idosos de Fortaleza. Tendo em vista os aspectos apresentados e a inexistência de informações, este estudo teve como objetivo avaliar, por meio de diferentes indicadores antropométricos, o estado nutricional dos idosos de Fortaleza.

\section{PROCEDIMENTOS METODOLÓGICOS}

Este é um estudo populacional, de base domiciliar, do tipo transversal, com coleta de dados primários. 0 estudo é constituído por indivíduos com 60 anos ou mais, de ambos os sexos e residentes habituais em domicílios particulares da cidade de Fortaleza/CE. Foram excluídos do estudo idosos em fase terminal de doença, idosos que apresentassem debilidade clínica grave e idosos que estivessem ausentes de Fortaleza por mais tempo que a pesquisa de campo naquele setor. A descrição detalhada do plano de amostragem deste estudo é apresentada no estudo de Menezes et al. ${ }^{18}$. De forma breve, o cálculo da amostra considerou o número de domicílios de Fortaleza. Para o dimensionamento do número de domicílios a serem visitados, foi adotado o modelo de amostragem estratificada proporcional, para o qual se estipulou $5 \%$ o erro amostral máximo admissível para os testes.

A seleção da amostra foi realizada de acordo com a metodologia utilizada pelo Instituto Brasileiro de Geografia e Estatística (IBGE), que divide as cidades em setores censitários. O plano de amostragem foi elaborado com base em técnicas de processos probabilísticos com estratificação dos bairros e setores censitários de Fortaleza, para garantir a representatividade da área territorial em que a pesquisa foi realizada.

As técnicas amostrais foram planejadas em múltiplas etapas, cuja seleção foi desenvolvida em estágios sucessivos. Inicialmente, foram selecionados, por meio de sorteio, os bairros. Em seguida, foram selecionados aleatoriamente os setores censitários em cada bairro sorteado. A identificação do domicílio foi realizada de forma sistemática. A identificação do primeiro domicílio ocorreu aleatoriamente, considerando como ponto de partida a esquina inicial do setor, em sentido horário. A cada domicílio selecionado, Foi saltado um número de domicílios, de acordo com cada setor censitário, e assim sucessivamente, a fim de obter-se melhor distribuição. Caso não residisse idoso na casa selecionada ou o mesmo não aceitasse (recusa) ou não pudesse (perda) participar do estudo, outro idoso era procurado no domicílio seguinte. No caso de residir mais de um idoso no domicílio, todos os idosos do domicílio foram convidados a participar do estudo.

\section{Avaliação antropométrica}

Foram analisadas as seguintes variáveis antropométricas: índice de massa corporal (IMC) (kg/ $\left.\mathrm{m}^{2}\right)$, dobra cutânea tricipital (DCT) $(\mathrm{mm})$ e circunferência muscular do braço (CMB) (cm).

Para o cálculo do Índice de Massa Corporal (IMC), que consiste na medida do peso corporal $(\mathrm{kg})$, dividido pela estatura $(\mathrm{m})$ elevada ao quadrado $(\mathrm{P} /$ $E^{2}$ ), foram mensurados a estatura e o peso com base nas técnicas propostas por Gordon et al. ${ }^{19}$. A DCT foi aferida, utilizando o compasso Lange $^{20}$. Para o cálculo da CMB, além da DCT, foi aferido, ainda, o perímetro do braço (PB), cuja aferição foi realizada de acordo com 
as técnicas de Callaway et al. ${ }^{21}$. A CMB foi calculada a partir dos valores de DCT e PB, utilizando a seguinte equação ${ }^{22}: \mathrm{CMB}=\mathrm{PB}-(\pi \times \mathrm{DCT})$.

Para análise do IMC, DCT e CMB, foram utilizados os valores descritos no estudo de Kuczmarski et al. ${ }^{2}$, apresentados em percentis, de acordo com sexo e grupo etário. Com base nos valores das variáveis antropométricas de cada idoso, definiu-se a seguinte adequação para IMC e DCT: insuficiente (s percentil 25), eutrofia (> percentil $25 \mathrm{e}<$ percentil 75) e excessivo ( $\geq$ percentil 75 ). Para CMB, definiu-se a seguinte adequação: eutrofia (> percentil 25) e desnutrição (s percentil 25). A partir dos diagnósticos obtidos com a análise das variáveis antropométricas, definiu-se a seguinte adequação do estado nutricional:

- Eutrófico - idoso, no qual as três variáveis antropométricas (IMC, DCT e CMB), simultaneamente, indicassem o estado de eutrofia, segundo os padrões adotados.

- Não eutrófico - demais idosos.

\section{Análise dos dados}

As informações estatísticas foram obtidas com o auxílio do aplicativo estatístico SPSS 10.0 e STATA 8.0.

Os indivíduos foram agrupados segundo sexo (masculino e feminino) e grupo etário (60-69 anos, $70-79$ anos e 80 anos ou mais). Foram calculadas as proporções de adequação (insuficiente, eutrofia, excessivo) das variáveis IMC e DCT. Para a CMB foram calculadas as proporções de indivíduos eutróficos e desnutridos. Foi realizado o teste quiquadrado $\left(\chi^{2}\right)$ para verificar a associação entre a adequação das variáveis antropométricas, sexo e grupo etário. Foram verificadas as proporções de idosos eutróficos e não eutróficos, em relação ao seu estado nutricional, assim como sua associação com sexo e grupo etário. Em todas as análises foi utilizado o nível de significância $\propto<5 \%$.

\section{Questões éticas}

Este estudo foi aprovado pelo Comitê de Ética em Pesquisa (COEP) da Faculdade de Saúde Pública/USP (protocolo de pesquisa $n^{\circ}$. 527). Os idosos receberam explicações a respeito do estudo e, ao concordarem em participar, assinaram o termo de consentimento livre e esclarecido, segundo Resolução $n^{\circ}$. 196, de 10 de outubro de 1996. No caso dos idosos, que por algum motivo, encontravam-se impossibilitados de assinar o termo de consentimento, foi solicitado ao responsável que o fizesse; caso este não estivesse no domicílio, foi realizada nova visita. Os idosos que se recusaram, não participaram do estudo.

\section{RESULTADOS}

Durante a seleção dos domicílios, houve 26 domicílios cujos idosos não aceitaram participar e 5 condomínios não autorizaram a entrada dos entrevistadores. No entanto, isso não comprometeu o plano amostral, visto a possibilidade que o mesmo oferecia de substituição imediata dos idosos que não pudessem ou não aceitassem participar da pesquisa.
Foram selecionados 385 domicílios para comporem a amostra deste estudo, onde foram entrevistados 483 idosos (68\% mulheres) residentes na cidade de Fortaleza. A média etária dos idosos foi 70,7 anos $(\mathrm{DP}=7,8)$, sendo 70,9 anos $(\mathrm{DP}=7,9)$ para mulheres e 70,3 anos $(D P=7,8)$ para homens.

\section{Avaliação antropométrica}

A tabela 1 mostra a distribuição dos idosos de acordo com a adequação do IMC, sexo e grupo etário. A prevalência de eutrofia foi de $47,3 \%$ entre o total de idosos. Proporções similares de eutrofia foram observadas entre os sexos (47,4\% para homens e $47,2 \%$ para mulheres). Os idosos do sexo masculino apresentaram menor prevalência de IMC com valor excessivo $(13,5 \%)$ quando comparados às mulheres $(21,9 \%)$. Foi verificada associação estatisticamente significativa entre adequação de IMC e sexo $(p=0,048)$. Na tabela 1, é possível observar, ainda, que o grupo etário de 60-69 anos foi o que apresentou maior proporção de idosos com valores de IMC insuficiente (36\%). O grupo 70-79 anos apresentou maior prevalência de eutrofia $(54,7 \%)$. A proporção de idosos com valores excessivos de IMC foi similar entre os grupos $60-69$ anos $(22,5 \%)$ e 80 anos ou mais $(22,2 \%)$. Não foi encontrada associação estatisticamente significativa entre adequação de IMC e grupo etário $(p=0,062)$.

Tabela 1. Distribuição dos idosos de Fortaleza, de acordo com adequação do IMC, segundo sexo e grupo etário.

\begin{tabular}{lcccc}
\hline \multirow{2}{*}{ Variáveis } & \multirow{2}{*}{ Categoria } & \multicolumn{3}{c}{ Índice de Massa Corporal (IMC) } \\
\cline { 3 - 5 } & & Insuficiente & Eutrofia & Excessivo \\
\hline Sexo* & & & \\
& Masculino & 39,1 & 47,4 & 13,5 \\
& Feminino & 30,9 & 47,2 & 21,9 \\
Grupo & & & & \\
etário & & & & \\
& $60-69$ & 36,0 & 41,5 & 22,5 \\
& $70-79$ & 31,5 & 54,7 & 13,8 \\
& 80 e + & 30,2 & 47,6 & 22,2 \\
& Total & 33,5 & 47,3 & 19,2 \\
\hline
\end{tabular}

* associação estatisticamente significativa entre os sexos.

Os valores da DCT mostram que $54,4 \%$ dos idosos foram considerados eutróficos. Os idosos do sexo masculino foram os que se apresentaram em maior proporção, com valores da DCT excessivos $(23,7 \%)$. As mulheres apresentaram maior prevalência de eutrofia (58\%) quando comparadas aos homens $(46,8 \%)$. Foi verificada associação estatisticamente significativa entre a adequação da DCT e o sexo $(p=0,039)$ (Tabela 2).

O grupo etário de 60-69 anos foi o que apresentou maior proporção de idosos com valores da DCT insuficientes $(28,4 \%)$. Os idosos com $70-79$ anos apresentaram maior prevalência de eutrofia $(58,6 \%)$ quando comparados aos idosos dos demais grupos etários. $\mathrm{O}$ grupo de idosos com 80 anos ou mais foi 
o que apresentou maior proporção de idosos com valores excessivos de DCT (30,1\%). Não foi encontrada associação estatisticamente significativa entre a adequação da DCT e grupo etário $(p=0,089)$ (Tabela 2).

Tabela 2. Distribuição dos idosos de Fortaleza, de acordo com adequação da DCT, segundo o sexo e o grupo etário.

\begin{tabular}{lcccc}
\hline \multirow{2}{*}{ Variáveis } & Categoria & \multicolumn{3}{c}{ Dobra Cutânea Tricipital (DCT) } \\
\cline { 3 - 5 } Sexo* & & & & \\
& & & & \\
& Masculino & 29,5 & 46,8 & 23,7 \\
& Feminino & 26,3 & 58,0 & 15,7 \\
Grupo & & & & \\
etário & & & & \\
& $60-69$ & 28,4 & 54,2 & 17,4 \\
& $70-79$ & 26,0 & 58,6 & 15,5 \\
& 80 e + & 27,0 & 42,9 & 30,1 \\
& Total & 27,3 & 54,4 & 18,3 \\
\hline
\end{tabular}

* associação estatisticamente significativa entre os sexos.

Na tabela 3, é possível observar a distribuição dos idosos, de acordo com a adequação da CMB segundo o sexo e o grupo etário. A desnutrição foi significativamente mais comum entre os homens que entre as mulheres $(p=0,000)$. As proporções de idosos desnutridos e eutróficos mostraram-se similares, para cada grupo etário. Os idosos do grupo 60-69 anos apresentaram maior proporção de idosos eutróficos $(51,7 \%)$. O grupo etário que apresentou maior proporção de idosos desnutridos foi o 70-79 anos $(50,3 \%)$. Não foi verificada associação estatisticamente significativa entre a adequação da $\mathrm{CMB}$ e o grupo etário $(p=0,916)$.

Tabela 3. Distribuição dos idosos de Fortaleza, de acordo com adequação da CMB, segundo o sexo e o grupo etário.

\begin{tabular}{cccc}
\hline \multirow{2}{*}{ Variáveis } & Categoria & \multicolumn{2}{c}{$\begin{array}{c}\text { Circunferência Muscular } \\
\text { do Braço (CMB) }\end{array}$} \\
\cline { 3 - 4 } Sexo* & & Desnutrição & Eutrofia \\
& & & \\
& Masculino & 66,5 & 33,5 \\
Grupo & Feminino & 40,6 & 59,4 \\
etário & & & \\
& $60-69$ & 48,3 & 51,7 \\
& $70-79$ & 50,3 & 49,7 \\
& 80 e + & 48,4 & 51,6 \\
& Total & 49,1 & 50,9 \\
\hline
\end{tabular}

* associação estatisticamente significativa entre os sexos.

Estado nutricional

A tabela 4 mostra a distribuição dos idosos de Fortaleza, de acordo com estado nutricional, segundo o sexo e o grupo etário. A maior parte dos homens $(83,9 \%)$ foi considerada não eutrófica, assim como maior parte das mulheres $(74,2 \%)$. Foi observada associação estatisticamente significativa entre o estado nutricional e o sexo $(p=0,018)$. O grupo etário de 80 anos ou mais foi o que apresentou maior proporção de idosos não eutróficos. O grupo 79-79 anos apresentou maior prevalência de eutrofia $(24,4 \%)$. Não houve associação estatisticamente significativa entre o estado nutricional e o grupo etário $(p=0,552)$.

Tabela 4. Distribuição dos idosos de Fortaleza, de acordo com o estado nutricional, segundo o sexo e o grupo etário.

\begin{tabular}{lccc}
\hline \multirow{2}{*}{ Variáveis } & Categoria & \multicolumn{2}{c}{ Estado nutricional } \\
\cline { 3 - 4 } & & Eutrófico & Não eutrófico \\
\hline Sexo* & & 16,1 & 83,9 \\
& Masculino & 25,8 & 74,2 \\
Grupo & Feminino & & \\
etário & & & \\
& $60-69$ & 22,5 & 77,5 \\
& $70-79$ & 24,4 & 75,6 \\
& 80 e + & 17,7 & 82,3 \\
& Total & 22,6 & 77,4 \\
\hline
\end{tabular}

* associação estatisticamente significativa entre os sexos.

\section{DISCUSSÃO}

Este estudo é parte do primeiro estudo populacional, de base domiciliar, realizado em Fortaleza, que envolve coleta de dados primários relativos à antropometria, consumo alimentar e nível sócio-econômico dos idosos. Sendo a amostra representativa da população estudada, as inferências obtidas neste estudo poderão ser extrapoladas para a população idosa de Fortaleza.

A condição nutricional do idoso tem sido estudada internacionalmente ${ }^{2,4,6,23}$. No Brasil, a situação não é diferente; no entanto, em menor escala, pesquisas com idosos vêm sendo realizadas, tanto em nível populacional ${ }^{3,4,10-13,17}$ como em grupos isolados ${ }^{12,25}$. Apesar dos diversos estudos com idosos desenvolvidos no Brasil, não foi encontrado na literatura estudo populacional, realizado no Nordeste, avaliando o estado nutricional de idosos, por conseguinte, em Fortaleza, o que torna a situação nutricional desse segmento da população desconhecida na maior parte da região.

Os valores de referência utilizados para verificar a adequação das variáveis antropométricas dos idosos, foram os descritos no estudo de Kuczmarski et al. ${ }^{2}$, que fornece informações obtidas a partir do NHANES III (Third National Health and Nutrition Examination Survey). O Expert Committee of the World Health Organization alerta para a necessidade de valores antropométricos de referência para idosos específicos por região e não recomenda a utilização de dados universais, ou seja, padrão de referência de determinado país, sendo utilizado pelos demais. No entanto, para os países que não possuem seus 
próprios padrões de referência ou pesquisas que os desenvolvam, o comitê sugere que sejam utilizados os valores provenientes do NHANES III. Infelizmente, o Brasil ainda não possui padrão antropométrico de referência nacional para idosos, dessa forma, estudos realizados com idosos, no Brasil, utilizam padrões internacionais ${ }^{8-10,13,24}$. Alguns estudos realizados no Brasil sugerem que os valores antropométricos da população de estudo, sejam utilizados como referência na avaliação de idosos ${ }^{11,12}$.

Quanto às variáveis antropométricas, algumas, por si, apresentam limitações. O IMC, indicador mundialmente utilizado na avaliação nutricional de idosos, principalmente em estudos populacionais, pode ter sua utilização restringida em indivíduos acamados ou cadeirantes, visto a necessidade de outras variáveis na estimativa do peso e estatura desses idosos. Além disso, a presença de edema poderia ocasionar resultados equivocados. A DCT, por ser uma medida sensível, torna-se mais suscetível a erros de medição, além da dificuldade por conta de diferenças na elasticidade e compressibilidade da pele. O PB, utilizado para o cálculo da $\mathrm{CMB}$, pode apresentar problemas na aferição e nos valores da medida, por conta da presença de edema e pela diferença na compressibilidade da pele. No entanto, há que se considerar que métodos mais precisos de avaliação nutricional (impedância bioelétrica, pesagem hidrostática, radioabsorciometria de feixes duplos DEXA) são caros e, na maioria das vezes, inviáveis para pesquisas populacionais, com base domiciliar. Diante das dificuldades com o método, cabe ao pesquisador tentar minimizar, ao máximo possível, falhas e limitações, investindo em treinamento, equipamentos adequados e utilizando o padrão de referência que mais se aproxime da população estudada.

$O$ índice de massa corporal (IMC) é um indicador do estado nutricional, mundialmente conhecido e utilizado em pesquisas populacionais, tendo em vista a disponibilidade e relativa facilidade em obtenção dos dados $^{26}$, bem como, sua relação com morbimortalidade. Valores de IMC acima dos valores considerados normais estão relacionados com aumento da mortalidade por doenças cardiovasculares e diabetes, enquanto que índices abaixo dos valores normais estão relacionados com aumento da mortalidade por câncer, doenças respiratórias e infecciosas ${ }^{27}$. Estudo realizado por Barreto et al. ${ }^{24}$, com idosos residentes em Bambuí/MG, utilizando o IMC como forma de verificar prevalência de obesidade e baixo peso, observaram que ambos foram associados a uma maior morbidade.

Para a avaliação da adequação de IMC deste estudo, foram considerados eutróficos os idosos com valores pertencentes aos percentis 25-75, que correspondem a um IMC que varia de $22,4 \mathrm{~kg} / \mathrm{m}^{2}$ a 30 $\mathrm{kg} / \mathrm{m}^{2}$ para homens e de $21,7 \mathrm{~kg} / \mathrm{m}^{2}$ a $30,8 \mathrm{~kg} / \mathrm{m}^{2}$ para mulheres. Troiano et al. ${ }^{28}$, realizando meta-análise para estimar a relação entre IMC e causas de mortalidade, sugerem como IMC adequado valores $>23 \mathrm{~kg} / \mathrm{m}^{2} \mathrm{e}<$ $28 \mathrm{~kg} / \mathrm{m}^{2}$, tendo em vista a existência de risco à saúde tanto para valores inferiores, como superiores. No estudo populacional de Marucci e Barbosa ${ }^{10}$, realizado com idosos do município de São Paulo, considerando os percentis 25 e 75 na distribuição dos valores de IMC, observa-se que para as mulheres os valores variam de $23,74 \mathrm{~kg} / \mathrm{m}^{2}$ a $30,36 \mathrm{~kg} / \mathrm{m}^{2}$ e para os homens de 22,5 $\mathrm{kg} / \mathrm{m}^{2}$ a $27,48 \mathrm{~kg} / \mathrm{m}^{2}$. A partir dos dados propostos por Perissinotto et al. ${ }^{29}$ para serem utilizados como padrões de distribuição antropométrica para a população idosa italiana, observa-se que ao considerar os percentis $25 \mathrm{e}$ 75 para IMC, os valores seriam de $23 \mathrm{~kg} / \mathrm{m}^{2}$ a $29,1 \mathrm{~kg} / \mathrm{m}^{2}$ para homens e $23,5 \mathrm{~kg} / \mathrm{m}^{2}$ a $31,2 \mathrm{~kg} / \mathrm{m}^{2}$ para mulheres. Dessa forma, observa-se que os valores utilizados como referência para este estudo se assemelham aos utilizados e/ou sugeridos em outros estudos.

A prevalência de idosos eutróficos foi similar entre os sexos (47,4\% homens e $47,2 \%$ mulheres). Tavares e Anjos ${ }^{17}$, analisando os dados da população idosa brasileira participante da Pesquisa Nacional sobre Saúde e Nutrição (PNSN 1989), encontraram que 61,8\% dos homens e $41,4 \%$ das mulheres eram eutróficos. No Nordeste, estes valores foram de $68,3 \%$ e 50,7\% para homens e mulheres, respectivamente; sendo essa a região que apresentou maior proporção de idosos com estado nutricional adequado, quando comparada às demais regiões. Como visto, o estudo de Tavares e Anjos $^{17}$ apresentou maior proporção de indivíduos eutróficos que este estudo, principalmente em relação aos homens; no entanto, essa diferença deve ser verificada com cautela, visto a diferença no padrão de adequação da variável, no plano de amostragem, no número maior de indivíduos provenientes das diferentes regiões, os quais apresentam diferenças sociais, econômicas e culturais.

Diferentemente deste estudo, de acordo com outros levantamentos realizados com idosos, a prevalência de eutrofia é, comumente, maior entre os homens ${ }^{9,13,17}$. Mastroeni ${ }^{13}$, utilizando o mesmo padrão de referência e ponto de corte deste estudo, registrou que $60 \%$ dos homens e $55 \%$ das mulheres encontravam-se eutróficos.

A prevalência de baixo peso foi superior à encontrada em outros estudos ${ }^{3,9,10,13,17}$ e ao verificar os dados por sexo, verificou-se que, à semelhança de outros estudos realizados com a população idosa brasileira, a prevalência de baixo peso foi maior entre os homens ${ }^{9,10,13}$.

Tavares e Anjos ${ }^{17}$ constataram que a prevalência de magreza entre os idosos estudados foi $8,8 \%$. Estudos recentes ${ }^{9,13}$ têm encontrado prevalências de baixo peso superiores às encontradas por Tavares e Anjos ${ }^{17}$, porém, inferiores às encontradas neste estudo. Barbosa ${ }^{9}$ registrou prevalência de $24 \%$ de baixo peso entre os idosos do município de São Paulo. Mastroeni ${ }^{13}$ verificou que $10 \%$ dos idosos de Joinville/SC apresentaram IMC abaixo do padrão de referência utilizado e que a maior proporção foi entre os homens (16\%) $\left(5,2 \%\right.$ entre as mulheres). Menezes ${ }^{8}$, em pesquisa realizada com idosos institucionalizados na cidade de Fortaleza, observou elevadas proporções de idosos com valores de IMC insuficientes, tanto para homens $(54,3 \%)$, quanto para mulheres $(47,9 \%)$. 
Essa maior proporção de idosos com IMC insuficiente, encontrada no estudo de Menezes ${ }^{8}$, em relação aos estudos de Tavares e Anjos ${ }^{17}$, Barbosa ${ }^{9}$ e Mastroeni ${ }^{13}$, deve-se, principalmente, à institucionalização, visto que estudos demonstram diferenças de IMC entre os idosos institucionalizados e não institucionalizados ${ }^{30,31}$, tendo o primeiro grupo valores menores.

Como visto, as prevalências de baixo peso na população idosa variam consideravelmente entre os estudos. No entanto, independente destas variações, essa parcela da população não pode ser desconsiderada, tendo em vista a elevada associação da magreza à mortalidade em idosos ${ }^{27,32}$.

Apesar da elevada prevalência de idosos eutróficos, as proporções de idosos com valores de IMC insuficientes e excessivos mostram a heterogeneidade do perfil nutricional da população estudada. Considerando que valores insuficientes, assim como excessivos de IMC caracterizam risco de morbimortalidade e que, ambos constituem maior prevalência neste estudo, é importante que haja monitoramento de ambas as situações, visto que o advento do aumento da obesidade em nossa população é recente, e que anteriormente a isso a preocupação maior era com a magreza. Sendo assim, será possível verificar a situação nutricional, assim como intervir de acordo com as particularidades e necessidades de cada população.

A dobra cutânea tricipital é uma variável indicativa da reserva de gordura subcutânea, amplamente utilizada em estudos antropométricos em idosos ${ }^{1,2,9,10,13}$. A proporção de mulheres eutróficas (58\%), deste estudo, foi superior à dos homens $(46,8 \%)$. No caso de valores de DCT insuficientes, a proporção foi maior entre os homens $(29,5 \%)$ quando comparados às mulheres $(26,3 \%)$. Mastroeni ${ }^{13}$ encontrou proporção similar de idosos com valores adequados de DCT entre os sexos ( $46,8 \%$ dos homens e $46,5 \%$ das mulheres) e, como neste estudo, maior proporção de homens apresentou DCT abaixo do adequado (32,9\%), quando comparados às mulheres $(9,4 \%)$. Fato que parece caracterizar os homens destes estudos, como tendo menor reserva de gordura, chegando a ser insuficiente.

Maior proporção de idosos com 80 anos ou mais apresentou medidas com valores excessivos para DCT, demonstrando que o grupo etário, ao qual o idoso faz parte, é um fator influenciador dessa maior reserva de gordura, tendo em vista que foi verificada associação estatisticamente significativa entre os grupos etários. O risco do excesso de peso para a saúde dos idosos, o qual pode ser indicado pelo maior acúmulo de gordura, ainda é incerto; o fato é que dados populacionais indicam que excesso de peso moderado em idosos está associado com baixa mortalidade. É sabido, ainda, que entre os idosos com 80 anos ou mais a magreza e perda de massa muscular pode ser um problema mais significativo que o excesso de peso moderado ${ }^{32}$. No entanto, há que se considerar que obesidade, além de resultar em perda de mobilidade ${ }^{27,32}$, consiste em fator de risco para o desenvolvimento de algumas doenças, como cardiovasculares ${ }^{33}$, e causa de mortalidade ${ }^{26}$.

A CMB é comumente utilizada na avaliação do estado nutricional de idosos, por ser uma medida sensível da massa muscular, possibilitando verificar a reserva protéica. Estudos mostram que os idosos tendem a ter menos massa magra que os adultos jovens, e que o efeito da idade é mais pronunciado depois dos 60 anos $^{27}$.

Apesar de apresentarem CMB maior que as mulheres, os homens deste estudo apresentaram maior proporção $(66,5 \%)$ de indivíduos desnutridos. Isso pode ser atribuído ao fato de, apesar de terem mais massa muscular que as mulheres, o depósito da maioria dos homens desta população não é suficiente para que os mesmos sejam considerados eutróficos.

Valores mais baixos de CMB em grupos etários mais elevados, assim como, a elevada prevalência de idosos desnutridos é preocupante, visto que, alterações musculares levam a manifestações clínicas, que podem influenciar de forma negativa na vida do idoso, pois a perda de massa muscular tem impacto sobre sua capacidade funcional. A força muscular, diretamente determinada pela quantidade de massa muscular, é uma medida preditora de independência e mobilidade em idosos ${ }^{34,35}$. Dessa forma, medidas indicadoras de massa muscular podem ser utilizadas em avaliação nutricional, auxiliando na detecção de riscos, de forma a garantir intervenções adequadas, melhorando a qualidade de vida do idoso.

Para a identificação do estado nutricional, foram utilizados, simultaneamente, os resultados da avaliação antropométrica pelo IMC, DCT e CMB, de forma a realizar o diagnóstico nutricional (eutrófico e não eutrófico), tendo em vista que indivíduos com bom estado nutricional apresentam adequado peso em função de sua estatura, adequada quantidade de reserva adiposa e muscular.

Avaliando as tabelas 1, 2 e 3, verifica-se que a proporção de eutróficos, a partir de cada variável, foi elevada $(47,3 \%, 54,4 \%, 50,9 \%$, respectivamente). Ao se avaliar o estado nutricional, a tabela 4 mostra diminuição dessa proporção de indivíduos eutróficos (22,6\%). Essa diminuição da proporção de idosos eutróficos deve-se, justamente, ao fato de, nesse caso, os idosos estarem sendo avaliados quanto ao seu peso para sua estatura, reserva de gordura e de massa muscular.

Esse modelo surgiu da necessidade dos autores de realizarem a avaliação do idoso de forma mais global e não apenas realizar o diagnóstico separadamente para cada variável. Quando se realiza diagnósticos separadamente, pode-se incorrer em sub ou superestimativas, como é o caso, por exemplo, dos idosos que são eutróficos quanto ao IMC, mas desnutridos, quanto à $\mathrm{CMB}$.

O modelo proposto neste estudo apresenta a limitação de categorizar os indivíduos apenas em eutróficos e não eutróficos; no entanto, a identificação dos indivíduos eutróficos já possibilita o conhecimento da situação nutricional da população. Apesar de, no caso dos não-eutróficos, os planos de ação serem diferentes, avaliar dessa forma, possibilita mensurar a magnitude do problema, seja ele por excesso ou 
por carência.

Esse modelo é uma tentativa de aprimorar a avaliação de idosos, por meio da utilização de variáveis antropométricas, visto que a mesma, apesar de suas limitações, representa importante instrumento de avaliação para pesquisas, sobretudo, pesquisas populacionais.

Como visto, as variáveis antropométricas são ferramentas amplamente utilizadas em estudos epidemiológicos para avaliar o estado nutricional de idosos. No entanto, estudos que utilizam mais de uma variável nessa avaliação, realizam os diagnósticos de forma separada, ou seja, um diagnóstico para cada variável antropométrica, não havendo assim, um diagnóstico nutricional global, envolvendo diferentes variáveis antropométricas.

Em geral, os resultados aqui apresentados confirmam estudos anteriores, os quais mostram a heterogeneidade da população idosa, em relação à sua situação nutricional. No entanto, há que se considerar que este estudo, por ser transversal, apresenta limitações, pois não permite avaliar a magnitude das alterações individuais nas características antropométricas aqui estudadas. Embora o estudo apresente essa limitação, os resultados aqui apresentados são coerentes com outros estudos transversais e longitudinais, os quais têm verificado resultados semelhantes quanto à influência da idade nas características antropométricas. Além disso, o seu desenho amostral foi suficiente para que os objetivos aos quais se propunha fossem alcançados, e por ser o primeiro estudo populacional que aborda aspectos nutricionais de idosos em uma capital do Nordeste, o mesmo pode ser útil como ponto inicial para novos estudos.

\section{CONCLUSÃO}

Conclui-se, dessa forma, que os idosos de Fortaleza apresentam estado nutricional vulnerável, tendo em vista as prevalências de inadequação, seja por excesso ou deficiência. Assim, torna-se necessária a promoção de vigilância nutricional ao idoso, em nível populacional e individual, com planos de ação diferenciados para idosos desnutridos e obesos. Essa atividade deverá contemplar a promoção e educação em saúde, com foco interdisciplinar, considerando, além do aspecto nutricional, os componentes sócioeconômicos e culturais a ele ligados.

\section{REFERÊNCIAS BIBLIOGRÁFICAS}

1. Suriah AR, Zalifah MK, Zainorni MJ, Shafawi S, Mimie Suraya S, Zarina N et al. Anthropometric measurements of the elderly. Mal J Nutr 1998;4:55-63.

2. Kuczmarski MF, Kuczmarski RJ, Najjar M. Descriptive anthropometric reference data for older Americans. J Am Diet Assoc 2000;100:59-66.

3. Campos MAG, Pedroso ERP, Lamounier JA, Colosimo EA, Abrantes MM. Estado nutricional e fatores associados em idosos. Rev Assoc Med Bras 2006;52(4):214-221.

4. Clausen T, Charlton KE, Holmboe-Ottesen G. Nutritional status, tobacco use and alcohol consumption of older persons in Botswana. J Nutr Health Aging 2006;10(2):104-110.

5. Barbosa AR, Souza JMP, Lebrão ML, Marucci MFN. Estado nutricional e desempenho motor de idosos de Saõa Pualo. Rev Assoc Med Bras 2007;53(1):75-79.

6. Sánchez-García S, García-Peña C, Duque-López MX, Juárez-Cedillo T, Cortés-Núñez AR, Reyes-Lópes $\mathrm{S}$. Anthropometric measures and nutritional status in a healthy elderly population. BMC Public Health 2007;7:2-10.

7. Ritchie CS, Burgio KL, Locher JL, Cornwell A, Thomas D, Hardin $\mathrm{M}$ et al. Nutritional status of urban homebound older adults. Am J Clin Nutr 1997;66:815-818.

8. Menezes TN. Avaliação antropométrica e do consumo alimentar de idosos residentes em instituições geriátricas da cidade de Fortaleza/Ceará. [Dissertação de Mestrado - Faculdade de Saúde Pública]. São Paulo: Universidade de São Paulo; 2000.

9. Barbosa AR. Estado nutricional e sua associação com força muscular, flexibilidade e equilíbrio de idosos residentes no município de São Paulo. [Tese de Doutorado - Faculdade de Ciências Farmacêuticas/ Faculdade de Economia, Administração e Contabilidade/ Faculdade de Saúde Pública]. São Paulo: Universidade de São Paulo; 2004.

10. Marucci MFN, Barbosa AR. Estado nutricional e capacidade física. In: Lebrão ML, Duarte YAO. SABE Saúde, Bem-estar e Envelhecimento - O projeto SABE no Município de São Paulo: uma abordagem inicial. $1^{\text {a }}$ ed. Brasília: Organização Pan-Americana de Saúde, 2003. p. 95-117.

11. Barbosa AR, Souza JMP, Lebrão, ML, Marucci MFN. Anthropometry of elderly residents in the city of São Paulo, Brazil. Cad Saude Publica 2005;21(6):1929-1938.

12. Menezes TN, Marucci MFN. Antropometria de idosos residentes em instituições geriátricas, Fortaleza, CE. Rev Saude Publica 2005;39(2):169-175.

13. Mastroeni MF. Estado nutricional e consumo de macronutrientes de idosos da cidade de Joinville, SC. [Tese de Doutorado - Faculdade de Saúde Pública]. São Paulo: Universidade de São Paulo; 2004.

14. Delarue J, Constans T, Malvy D, Pradignac A, Couet C, Lamisse F. Anthropometric values in an elderly French population. Brit J Nutr 1994;71:295-302.

15. Ramos LR, Toniolo JN, Cendoroglo MS, Garcia JT, Najas MS, Perracini M et al. Two-year followup study of elderly residents in S. Paulo, Brazil: methodology and preliminary results. Rev Saude Publica 1998;32(5):397-407.

16. Coelho Filho JM, Ramos LR. Epidemiologia do envelhecimento no Nordeste do Brasil: resultados de inquérito domiciliar. Rev Saude Publica 1999;33(5):445-453.

17. Tavares EL, Anjos LA. Perfil antropométrico da população idosa brasileira. Resultados da Pesquisa Nacional sobre Saúde e Nutrição. Cad Saude Publica 1999;15(4):327-333.

18. Menezes TN, Lopes FJM, Marucci MFN. Estudo domiciliar da população idosa de Fortaleza/CE: aspectos metodológicos e características sócio-demográficas. Rev Bras Epidemiol 2007;2(10):168-177.

19. Gordon CC, Chumlea WC, Roche AF. Stature, recumbent length, and weight. In: Lohman TG, Roche AF, Martorell $\mathrm{R}$. Anthropometric standardization reference manual. Champaign, Illinois: Human Kinetics Books, 1988.

20. Harrison GG, Buskirk ER, Carter JEL, Johnston FE, Lohman TG, Pollock ML, Roche AL, Wilmore J. Skinfold thicknesses and measurement technique. In: 
Lohman TG, Roche AF, Martorell R. Anthropometric standardization reference manual. Champaign, Illinois: Human Kinetics Books, 1988.

21. Callaway CW, Chumlea WC, Bouchard C, Himes JH, Lohman TG, Martin AD et al. Circumferences. In: Lohman TG, Roche AF, Martorell R. Anthropometric standardization reference manual. Champaign, Illinois: Human Kinetics Books, 1988.

22. Gurney JM, Jelliffe DB. Arm anthropometry in nutritional assessment: nomogram for rapid calculation of muscle circumference and cross-sectional muscle and fat areas. Am J Clin Nutr 1973;26:912-915.

23. Arroyo $P$, Lera L, Sánchez H, Bunout D, Santos JL, Albala C. Indicadores antropométricos, composición corporal y limitaciones funcionales em ancianos. Rev Med Chil 2007;135:846-854.

24. Barreto SM, Passos VMA, Costa MFFL. Obesity and underweight among Brazilian elderly. The Bambuí Health and Aging Study. Cad Saude Publica 2003;19(2):605-612.

25. Marucci MFN. Aspectos nutricionais e hábitos alimentares de idosos matriculados em ambulatório geriátrico. [Tese de Doutorado - Faculdade de Saúde Pública]. São Paulo: Universidade de São Paulo; 1992.

26. Bannerman E, Miller MD, Daniels LA, Cobiac L, Giles LC, Whitehead C, Andrews GR, Crotty M. Anthropometric indices predict physical function and mobility in older Australians: the Australian Longitudinal Study of Ageing. Public Health Nutr 2002;5(5):655-662.

27. World Health Organization. Physical status: the use and interpretation of anthropometry. Geneva; 1995. (WHO - Technical Report Series, 854).

28. Troiano RP, Frongillo EA, Sobal J, Levitsky DA. The relationship between body weight and mortality: a quantitative analysis of combined information from existing studies. Int J Obes Relat Metab Discord 1996;20:63-75.

29. Perissinotto E, Pisent C, Sergi G, Grigoletto F, Enzi G. Anthropometric measurements in the elderly: age and gender differences. Br J Nutr 2002;87:177-186.

30. Mowe M, Bohmer T. Nutrition problems among home-living elderly people may lead to disease and hospitalization. Nutr Rev 1996;54(1):22-24.

31. Roubenoff R, Giacoppe J, Richardson S, Hoffman PJ. Nutrition assessment in long-term care facilities. Nutr Rev 1996;54(1):S40-42.

32. van Baak MA, Visscher TLS. Public health success in recent decades may be in danger if lifestyles of the elderly are neglected. Am J Clin Nutr 2006;84:1257-1258.

33. Villareal DT, Miller BV III, Banks M, Fontana L, Sinacore DR, Klein S. Effect of lifestyle intervention on metabolic coronary heart disease risk factors in obese older adults. Am J Clin Nutr 2006;84:317-1323.

34. Frontera WR, Hughes VA, Fielding RA, Fiatarone MA, Evans WJ, Roubenoff. Aging of skeletal muscle: a 12-yr longitudinal study. J Appl Physiol 2000;88:1321-1326.

35. Landers KA, Hunter GR, Wetzstein CJ, Bamman MM, Weinsier RL. The interrelationship among muscle mass, strength, and the ability to perform physical tasks of daily living in younger and older women. J Gerontol 2001;56:B443-B448.

\section{Endereço para correspondência}

Tarciana Nobre de Menezes

Avenida das Baraúnas, 351. Campus I, Prédio dos Mestrados (NEPE). $2^{\circ}$. andar.

Recebido em 04/04/08

Bodocongó

CEP. 58109-753. Campina Grande/PB.

Revisado em 06/05/08

E-mail: tnmenezes@yahoo.com.br 